\title{
An Interactive User Interface for Text Display
}

\author{
Hyungsuk Ji and Hyunseung Choo \\ School of Information and Communication Engineering, \\ Sungkyunkwan University, Korea \\ jihyungsuk@skku.edu, choo@ece.skku.ac.kr
}

\begin{abstract}
Studies on the effect of text width on readability have encouraged the use of fixed text-width web/electronic text design. The drawback of this type of design is the loss of users' interactivity with regard to text modification. In this paper, we investigate the web design of the world's top 100 websites and present an alternative interactive user interface for text display.
\end{abstract}

Keywords: Readability, Web design, line length, text presentation.

\section{Introduction}

A great number of texts are read nowadays on computer screens. With the increase in the amount of web-based texts, it is becoming increasingly important to develop a convenient user interface that can enhance the efficiency and reduce the fatigue when reading via the web 1]. Readability, among others, is one of the most important factors that affect efficiency and work fatigue, and it is closely related to the text width or line length of the text.

The research on readability found that the largest acceptable text width for printed materials is around $90 \mathrm{~mm}$, with some varying results. With regard to the computer screen environment, the average text width preferred by the subjects on the computer screen was found to be around $100 \mathrm{~mm}$ [23]. An experiment measuring the number of characters showed similar results of 55 characters per line (cpl) 2] and 55-70 cpl [3. Based on these studies, web designs with a specific fixed text width were recommended [34. Influenced by these kinds of studies, a myriad of web pages are now designed to meet conditions for maximum readability, and the majority of them have fixed text width format.

While general consensus was found with regard to the best readability and line length in the above experiments, there have always been some inconsistent results. First, the similar readability was found irrespective of the line length as far as the line length does not exceed a certain limit 2]. Second, the optimum line length that corresponds to optimum readability differed depending on researchers and experiments [2]3].

In this study, we propose an alternative user interface that restores the interactive text-width-selecting feature without going back to the first generation web document style. 


\section{Web Design on Text Display}

The following web design history is a classification of the layout, or the controllability of the text width in web pages. These are based on the period where each characteristic feature was used effectively, and not at the point where the feature was first introduced 1

First Generation. The first generation web design was characterized by its simplistic layout. The text width was adjustable freely by dragging the borders of windows with the mouse or by clicking the restore/maximize button.

Second Generation. With the adaptation of 'table tags', partial restrictions were imposed on users' adjustability of text width.

Third Generation. The frame was introduced: the menu and the main content were separated. Adjusting the width of the menu and the main contents was possible and users had the ability to hide menus by minimizing their width.

Fourth Generation. With the practical use of Cascading Style Sheets (CSS), web pages became more flexible from a designer's point of view and more rigid from a user's point of view. Frames disappeared and it no longer became possible for users to remove or hide menus.

In the first generation, the adjustability of the text width provided web pages with the most interactive and free text format as far as text width modification was concerned. This feature was so typical that even today almost all word processors call "dynamic text width layout" "web page layout". However, influenced by the studies on readability and line length and by other design requirements, most web pages today have fixed text width and it is impossible for users to adjust the text width.

To quantify these trends, we investigated the world's top 100 websites as chosen by Alexa. The results showed that only 11 web pages have a dynamic text width format and about $90 \%$ of world's top 100 websites use fixed text width web pages 2 Considering the still permitted personalizable options such as font style, font size, color and global window size of web pages, it is quite surprising that the most popular web designs do not allow the slightest flexibility with respect to the text width. This rigidity of web page design has resulted in losing one of the most fundamental web pages' features and reducing user's active interaction ability with the computer.

\section{Proposed Interface}

For 11 websites where dynamic text width modification is allowed, resizing global window's (web browser's outer window's) width is required to get the desired

${ }^{1}$ CSS was first proposed in November 1995, which was virtually in the same period as the previous two (Table was first proposed in March 1995 and Frame September 1995).

2 The 11 websites are: *rkut.com *ikipedia.org *mazon *otolog.com *mdb.com *apidshare.de $*_{\text {andex.ru }}^{*}$ c2.com $*_{\text {ourceforge.net }}^{*}$ ama.ru $*$ igg.com (The first letter is marked out for each item.). 

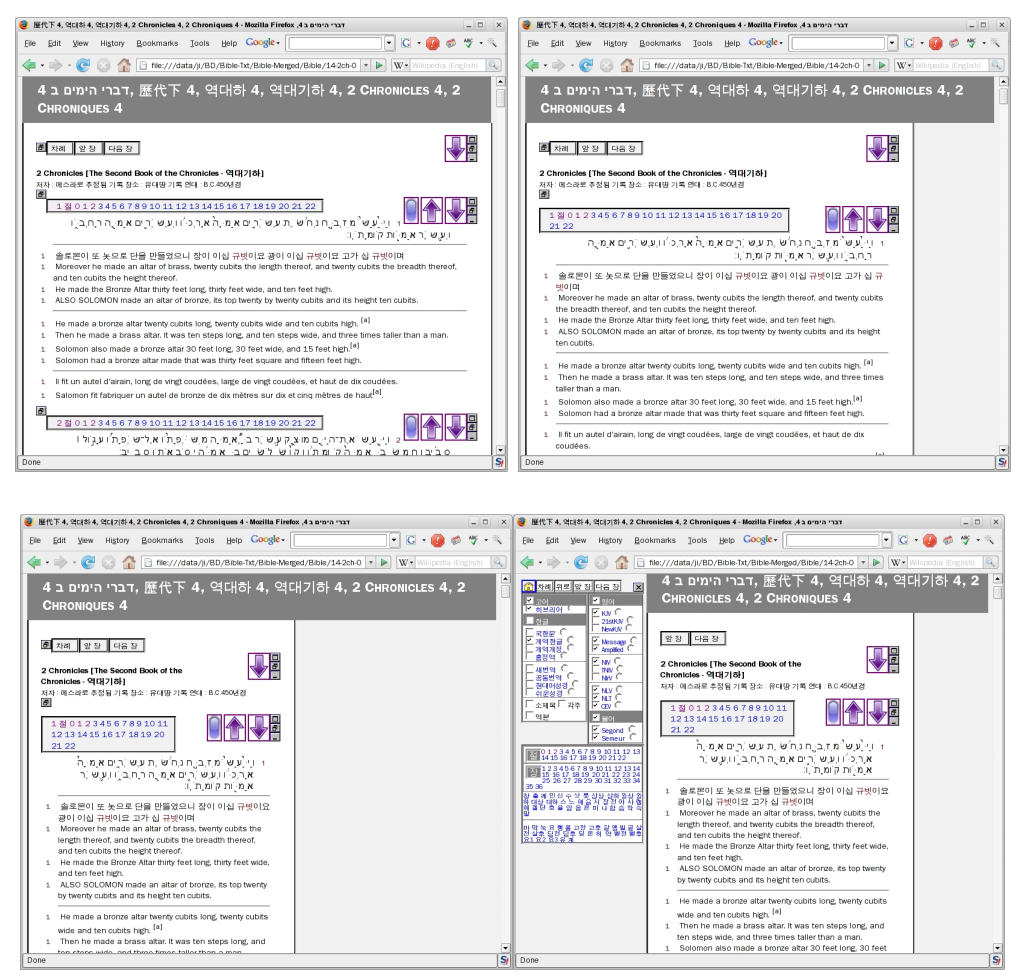

Fig. 1. The text width can be adjusted easily with a single mouse click

text width. This results in two difficulties. First, in narrowing global windows width, users lose access to the far right menus/tabs, unless the menus/tabs are detached from the web browser. Second, after narrowing text width, restoration is necessary to view other web pages. Since web page reading involves frequent changes from one website to another, this alternation is by no means convenient. Further, some websites have no limit on line length, which obliges users to resize their browser windows for better reading.

It is still important to allow users to personalize web text width. The results of line-length related studies show that the optimal line length is not identical for every user and considerable differences exist depending on the particular study group 35. Thus, providing users with interactive text-width modification functionality is important in order to provide users with more personalized control over the text format. In particular, in conducting specific textual study like analyzing multitext, such an interface would be of benefit. Multitext analysis study requires both normal reading and comparing two or more adjacent lines, and two types of text-width are necessary for the fulfillment of the task (unlike normal reading, it is preferable to have a greater line length for line-by-line comparison).

We propose a user interface that ensures users' interactive control in adjusting text format and provides a novel scrolling functionality for reading documents. 
Special buttons were placed top right of the documents so that users can adjust their contents' width by a simple single click with the mouse (Fig. 1). The button was designed without the use of mouse dragging since the latter requires non-trivial concentration and attention. We propose (1) two different text-width formats independent of global windows size and (2) a dynamic format, whose width is dependent on global window size.

The texts in Fig. 1 are parallel verses of the Bible, which were constructed automatically using the program 'multitext organizing model' that we developed in parallel with this study. In these multitext documents, the verses having the same verse number are grouped together and the arrow buttons in Fig. 1 allow users to bring the head of these groups on top of the window, ensuring the constant positioning of the aligned elements.

Hornbæk and Frøkjær reported that users prefer a fisheye interface, where unimportant texts are reduced in size, or presented as unreadable, to a linear interface, where no such distortions are implemented 6. One drawback of such a reduced size form is the amount of unimportant text cannot be known. Our proposed scrolling method can be useful in such a case, since it is possible both to keep full readable length of unimportant text, and to skip quickly over a portion of text. An extra functionality was implemented that enables users to select or remove certain portion of texts or left-side menu (the third and the fourth figure in Fig. 1). These features were developed using JavaScript and CSS and can be readily implemented in existing web documents.

Acknowledgments. This research was supported by the Ministry of Information and Communication, Korea under the ITRC, IITA-2006-(C1090-0603-0046).

\section{References}

1. Corry, M.D., Frick, T.W., Hansen, L.: User-centered design and usability testing of a web site: An illustrative case study. Educational Technology Research and Development 45(4) (1997) 65-76

2. Dyson, M.C., Haselgrove, M.: The influence of reading speed and line length on the effectiveness of reading from screen. International Journal of Human-Computer Studies 54(4) (2001) 585-612

3. Ling, J., van Schaik, P.: The influence of font type and line length on visual search and information retrieval in web pages. International Journal of Human-Computer Studies 64(5) (2006) 395-404

4. Davidov, A.: Computer screens are not like paper: typography on the web. In Sassoon, R., ed.: Computers and Typography. Volume 2. Intellect Books, Bristol, UK. (2002) 21-40

5. Mills, C.B., Weldon, L.J.: Reading text from computer screens. ACM Comput. Surv. 19(4) (1987) 329-357

6. Hornbæk, K., Frøkjær, E.: Reading patterns and usability in visualizations of electronic documents. ACM Trans. Comput.-Hum. Interact. 10(2) (2003) 119-149 